\title{
Facilitating User Involvement in Product Design Through Virtual Reality
}

\author{
J.P. Thalen and M.C. van der Voort
}

Additional information is available at the end of the chapter

http://dx.doi.org/10.5772/48602

\section{Introduction}

The product development process $(P D P)$ generally involves a sequence of gathering requirements, conceptual design, engineering, manufacturing and finally a market release. Successful product development depends on collaboration and communication between stakeholders (e.g. designers and engineers, but also end-users, marketeers or managers) throughout the phases in the development process. Tools that support this communication and collaboration primarily focus on internal communication. For instance, sketches and drawings facilitate communication between designers, CAD models facilitate communication between engineers and presentations or reports are used for communication between departments. Supporting external communication (i.e. communication between people inside the development process and people outside the process) is more challenging because external stakeholders (such as end-users or customers) are usually not trained in being involved in a PDP.

This chapter describes how Virtual Reality (VR) can facilitate the involvement of external stakeholders in the PDP. Stakeholder involvement can improve the information quality and quantity; end-user feedback for instance facilitates concept generation and selection, or identifies usability issues in an early stage. However, with only limited design information available it is difficult to provide stakeholders with a clear presentation of a product concept and future use context. We therefore propose to use VR technologies to create realistic concept representations in the early stages of the design process. VR technologies create an alternative reality in which worlds, objects and characters can be experienced that may not yet be available in reality. As such it allows stakeholders to not only see the future product (which could also be achieved with a concept sketch or mockup), but also experience the product and the interactions with its use context. 
In this chapter we first elaborate on stakeholder involvement in the PDP, and highlight the importance of involving future end-users in particular. We also depict some of the characteristics that make VR suitable for facilitating stakeholder involvement. The main body of the chapter comprises a set of industrial case studies that illustrate how VR can be applied to achieve different levels of user engagement in various phases of the PDP. This overview and comparison of case studies leads to conclusions regarding the selection of appropriate VR techniques for specific types of user involvement in product design.

\section{Background}

Traditionally, the PDP is referred to as a problem solving activity in which the result is determined by a series of technical decisions $[1,2]$. Recently, the product design process is more and more considered to be a group activity in which communication and collaboration between stakeholders plays a central role [3,4].

The importance of including these stakeholders, and in particular potential users, in the design process becomes clear when one considers the task-artefact cycle [5]. New products are designed to fulfill a certain task or activity, i.e. solve the signaled problem. However, the introduction of the new product will in return influence the identified problem. New problems may arise as well as new opportunities may be identified. As [6] explains: 'Design is always indeterminate, because design changes the world within which people act and experience' (p. 48). To support a continuous iterative design approach, a close and timely involvement of end-users in the design process is therefore required.

\subsection{Facilitating user involvement}

To successfully include end-users, a design process needs to have appropriate facilitating characteristics. In [7] the following set of conditions for effective and efficient participation of end-users is proposed.

- A direct and explicit communication between designer and end-user needs to be established. The way of communication should minimise the chance of misinterpretation on either side.

- End-users should be enabled to have a realistic interaction with the design information. They should be able to reliably assess the exact functioning and experience of the design under a wide range of circumstances.

- End-users should be enabled to reliably become conscious of and assess the consequences of design decisions. Consequences of design decisions should be made explicit and presented in a manner that is comprehensible regardless participant's training or discipline.

VR potentially provides solutions meeting all three conditions for successful user involvement. It supports a realistic interaction with design information: design information is presented in a way that is comprehensible regardless discipline or 
training, whereas consequences of design choices can be experienced rather than imagined [8]. The latter is especially beneficial when stakeholders (e.g. end-users) are unfamiliar with the product to be designed or with the technology that is suggested to be incorporated. The actual experience of design information and consequences will sincerely improve the reliability of the input provided by stakeholders to the design process. Furthermore, by using VR simulation, misunderstandings between human actors are less likely to occur compared to when using more abstract or symbolic representations of design information (such as natural language, sketches and CAD drawings). Another benefit of using VR simulation is that it eliminates the necessity to make physical prototypes. It not only saves money and time, but also allows for evaluation of candidate designs in an earlier phase of the design process [9]. Evaluation of candidate designs under dangerous or rare use circumstances is furthermore supported without compromising safety or efficiency.

While VR in principle meets all the proposed conditions, it is argued that VR is rarely a solution on its own. Integrating VR technologies with the PDP requires supporting principles to 'embed' the technology in the design process. In our case studies we primarily make use of scenarios and gaming techniques to do so.

Scenarios are explicit descriptions of hypothetical events concerning a product during a certain phase of its life cycle [10]. A scenario can be expressed by displaying a prototype (either real or virtual) in an environment (either real or virtual). Within design processes, scenarios are used to address problems, needs, constraints and possibilities. Like VR, the use of scenarios facilitates explicit communication of design information among involved stakeholders.

Gaming is generally described as a play with props following specific rules and often with an element of competition between players and decided by chance, strength, skill or a combination of these [11]. Within design, playing games is a way to generate ideas in a situated and participative way. Due to rapid developments in the gaming industry, the trend to use games as a design tool no longer means just board games and card games, but also computer games. Within a (computer) game, information is simultaneously generated, represented and evaluated. Therefore, design iterations can be performed very quickly. Furthermore, playing games helps to develop a common language between designers and users [12].

The majority of the case studies presented in this chapter use scenarios that were explicitly defined to serve as a frame of reference, whereas gaming techniques were added to trigger user participation.

\section{Framework}

This section introduces the framework through which the VR applications are presented and compared. The framework is based on the work of [13], who presents an overview of user involvement in product design methods. Each of our case studies will be positioned 
according to two dimensions, namely the design phase in which it is used and the intended level of user engagement.

The first dimension of the proposed framework represents the phase in the design process in which VR is applied. VR applications in product development (e.g. virtual prototyping, virtual assembly or virtual manufacturing) traditionally rely on CAD systems and consequently focus on advanced stages of the PDP, such as the engineering and manufacturing phase [14] for a survey). In this chapter however, we focus on the early stages (also known as the fuzzy front-end) of the PDP. The lack of concrete information in this stage of the PDP is challenging for designers, but also allows end-users to still have considerable impact on design decisions. The case studies will illustrate how the benefits of VR mentioned in the previous section facilitate various design activities in these early stages. To further characterise the case studies, the first two phases of the original framework (specification and conceptual design) are split into a specification phase, a concept generation phase and a concept evaluation phase. This allows for a differentiation between generative and evaluation applications that take place in the concept development phase.

The second dimension that is used to characterise the case studies is defined as the level of user engagement. User engagement is related to the extent to which a user is involved or participating in the design process. Interviews and surveys require a low level of user engagement, as the actual product development is on on behalf of the users (referred to as 'design for' in the original framework). A higher level of user engagement is achieved when users are allowed to try, evaluate and/or select proposed product concepts. This is referred to as 'design with' in the original framework. The highest level of user engagement is achieved through co-design activities, in which users actually generate product concepts themselves. This is referred to as 'design by' in the original framework.

Both dimensions have a continuous scale, meaning that there is no strict border between phases in the design process or level of stakeholder involvement. Some of the cases we present comprise an integrated design approach that spans the entire product development process, while other cases focus on a particular phase in the process.

\section{Case studies}

The case studies presented in this section originate from various research projects in which industrial partners participate by providing a design case. Consequently, there is a wide variety in application domains, ranging from automotive design and consumer electronics to healthcare and mechanical engineering. Furthermore, the industrial partners range from large multinationals to small or medium sized enterprises (SMEs). The involved industrial partners only had experience in the application of VR in the later design stages. Main reason was the unawareness of the involved industrial partners of the potential use of VR in the early stages of design; VR is associated with CAD applications, expensive user interfaces and technically complex tools. Showing demonstrations of early stage VR applications (often 
implemented using off-the-shelf technology) triggered the participants to explore new opportunities.

Each case study is placed in the framework according to the dimensions introduced in the previous section, as shown in Figure 1. As mentioned in the previous section, several case studies span multiple design phases. The detailed case study descriptions elaborate on this by explaining how some applications can indeed be useful across several design phases. Furthermore, the application context, technical implementation and the specific contribution to user involvement is explained.

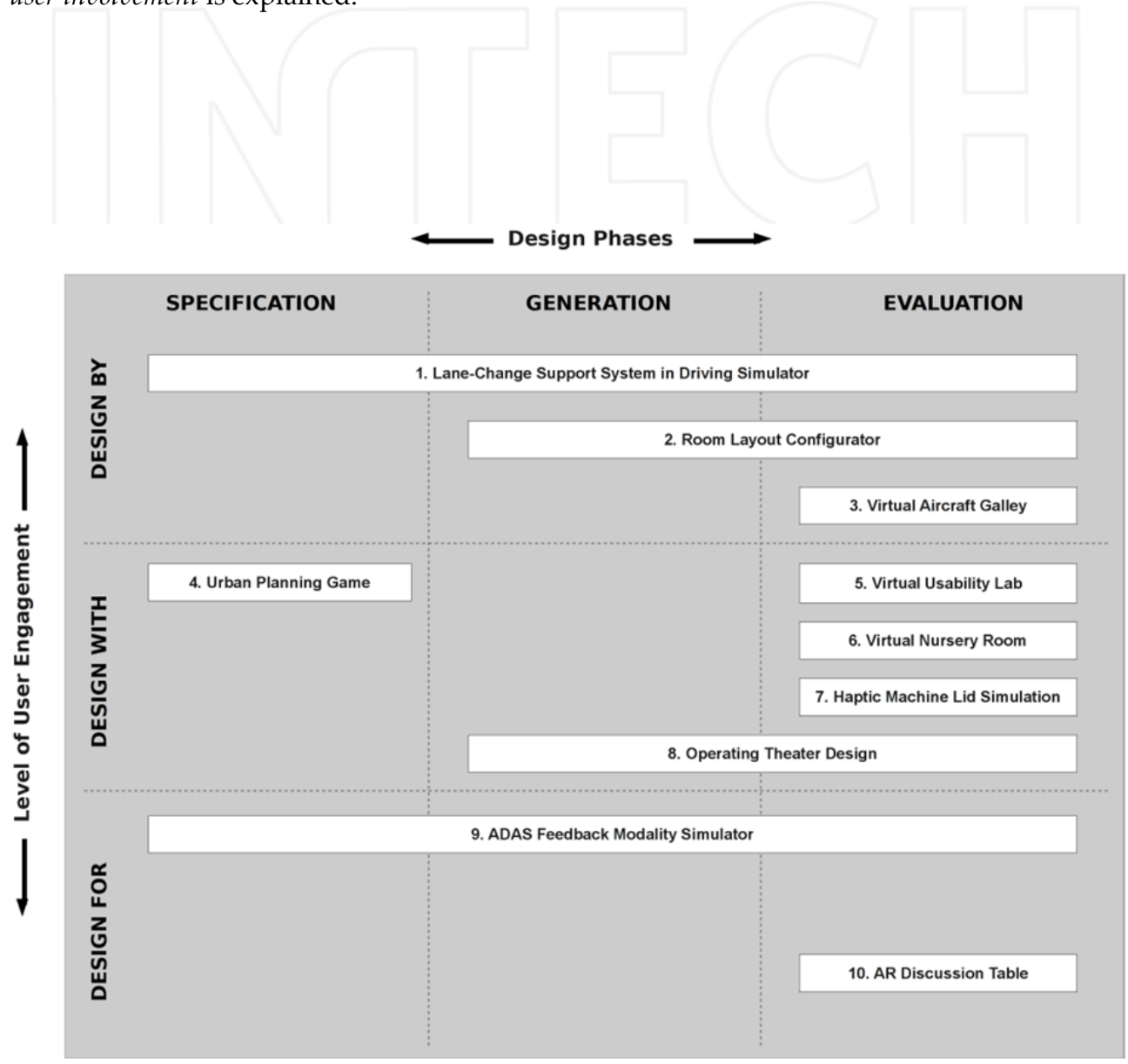

Figure 1. The framework in which case studies are positioned according to their place in the product development process and the achieved level of user engagement. 


\begin{tabular}{|l|l|l|}
\hline & Application Brief & $\begin{array}{l}\text { Application } \\
\text { Domain }\end{array}$ \\
\hline 1 & $\begin{array}{l}\text { A configurable user interface for lane-change systems, connected to an immersive } \\
\text { driving simulator. }\end{array}$ & Automotive \\
\hline 2 & $\begin{array}{l}\text { An interactive virtual room connected to a multi-touch input table to discuss room } \\
\text { layouts and visualise them in real-time. }\end{array}$ & Healthcare \\
\hline 3 & $\begin{array}{l}\text { An interactive virtual model of an aircraft galley, used to act-out scenarios and } \\
\text { identify and discuss innovative concepts. }\end{array}$ & Aerospace \\
\hline 4 & $\begin{array}{l}\text { A serious game implemented on a multi-touch table to facilitate group decisions } \\
\text { and visualise future urban planning scenarios. }\end{array}$ & Urban Planning \\
\hline 5 & $\begin{array}{l}\text { A virtual office environment in which current and future workflows can be } \\
\text { experienced and evaluated. }\end{array}$ & Consumer \\
Electronics
\end{tabular}

Table 1. Brief descriptions of the ten case studies and their application domains.

\subsection{Lane change support system in driving simulator}

Application Context - To investigate user preferences for a new lane change support system, an immersive driving simulator was developed [15]. A lane change support system helps a driver to safely change lanes on a highway, for instance by warning the driver about vehicles in a blind spot. The driving simulator allowed end-users to configure the user interface of this support system themselves, for instance by setting the level and type of support. In this case study a group of participants was asked to configure their ideal interface and evaluate it in a simulated traffic scenario.

Technical Implementation - The driving simulator uses a physical mockup of a car, positioned in front of a large semi-spherical display. The car mockup is equipped with configurable dashboard displays, audio speakers and actuators to provide various output modalities including visual cues, auditory cues and tactile feedback (see figure 2). The dashboard also features a Wizard-like application that guides participants through the dashboard configuration options. 


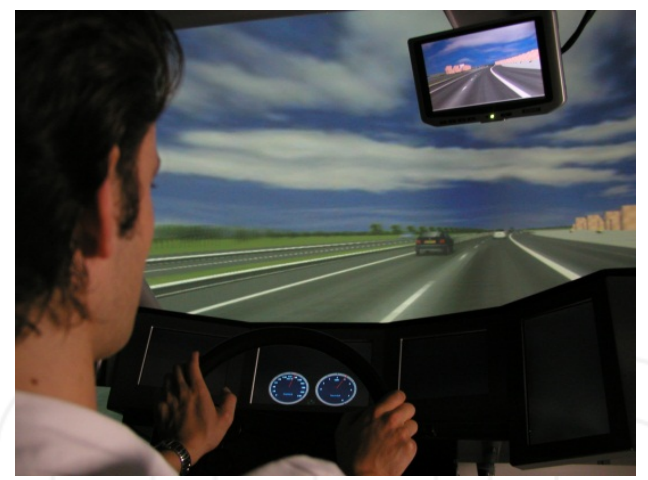

Figure 2. The driving simulator used for testing lane-change support systems, also showing the configurable dashboard.

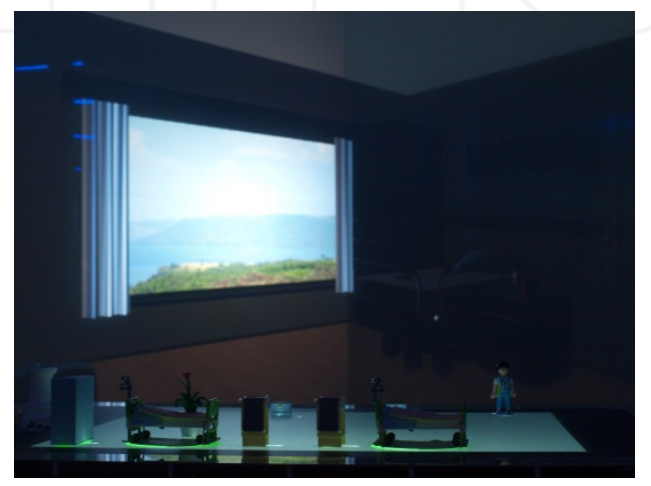

Figure 3. The room layout configurator. The foreground shows the surface table with tangible objects. The background shows the wall display with the virtual room.

User Involvement - The approach that was used in this case study supports designers in determining stakeholders' preferences and finding the best compromise between those preferences. This resulted in a hierarchy of information that is a detailed, consistent and reliable image of users' preferences. The hierarchy provides the designer insight in the relationship between user characteristics, use circumstances and desirable or undesirable product characteristics. The evaluation revealed that the new participatory design approach is viable in the sense that people understood their role in the design process, were able to perform the specified activities, and that these activities yielded actual results. Users were enabled to directly express their preferences and realistically experience the consequences of their decisions. Moreover, determining users' preferences has happened while immersing them in the relevant context, but without putting them in dangerous situations.

\subsection{Room layout configurator}

Application Context - This case study was carried out in cooperation with a design department particularly interested in ambient experience design [16]. Designers currently use 
physical replica rooms to test different lighting and sound concepts in various contexts. These physical rooms are not very flexible and cost efficient; a special room has to be built depending on the theme that is under investigation (e.g. hospital rooms, hotel rooms or living rooms). In this case study a VR application was developed to help designers with easily creating room layouts and evaluating the ambient experience.

Technical Implementation - The application consists of a Microsoft Surface Table (a table-size horizontal multi-touch display) connected to a large projection wall (see figure 3 ). The wall shows a 'first-person' view of a virtual room. The arrangement of furniture, as well as the first-person camera perspective can be controlled using tangible objects located on the Surface Table that are traced using visual tags underneath each object.

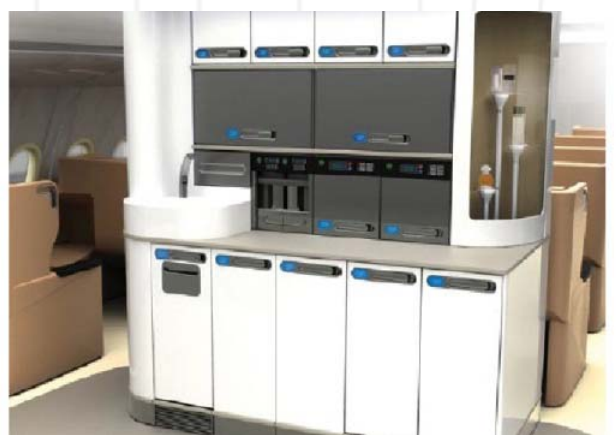

Figure 4. The virtual aircraft galley.

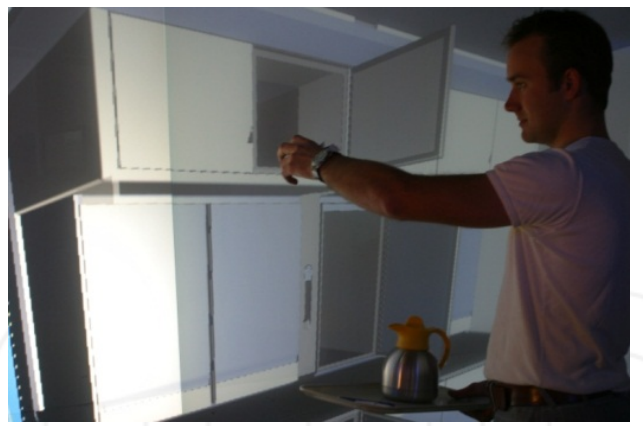

Figure 5. The virtual aircraft galley allows users to interact with the virtual model.

User Involvement - The setup is designed to facilitate multidisciplinary group meetings. The Surface Table interface enables a group (e.g. designers, end-users, experts, etc.) to collaboratively compose and review the layout of the room, regardless of the background and expertise of the group members. Each participant has an equal share in determining the room layout and everyone is able to explain his/her perspective on the design case. While group participants are able to create and change the layout of the room, the application primarily targets concept evaluation tasks; product concepts can be demonstrated and 'experienced' in different settings. 


\subsection{Virtual aircraft galley}

Application Context - In [17] a synthetic environments consultancy approach is proposed that supports small and medium-sized enterprises in identifying VR opportunities for their product development process. To evaluate this approach, a case study for a design agency was performed that concerned the redesign of an aircraft galley. The redesign aims to achieve weight reduction, extra chairs in the cabin, easy exchangeable equipment and improved ergonomics. The consultancy approach guided the design agency through a series of workshops in which the designers identified potential bottlenecks in their development process. The approach used scenarios extensively to communicate the findings of these workshops between the researcher and the designers; for instance to describe potential implementations of VR in the design process.

Technical Implementation - In the end, the design agency decided to use an interactive walkthrough model of the aircraft galley, projected on a large mutli-touch wall display (see figures 4 and 5). The application for instance allowed designers to virtually walk through the galley, and open doors or trays. The space in front of the multi-touch wall display is furnished such that it represents the space in the galley.

User Involvement - The case study illustrates how a 3D interactive visualisation can support multidisciplinary meetings for the design agency. The setup facilitates scenario visualisation but also triggers more interactive behavior in the group; session participants are encouraged to step in and show other participants what they have in mind in a particular situation.

\subsection{Urban planning game}

Application Context - 'Serious Games' facilitate decision making processes concerning complex problems. Urban planning is such a complex problem; developing cities, countries or regions is bound to involve many stakeholders and has many physical variables and constraints. This case study features the design of a serious game targeting the development of new city neighborhoods.

Technical Implementation - The resulting application is a multi-player collaborative serious game played on a Microsoft Surface Table. The table displays a top view of a city in which the neighborhood is to be developed (see figure 6). Game participants use tangible objects (e.g. bricks representing houses, trees, buildings, etc.) to modify the city design. The table responds to the bricks by immediately showing the results of a proposed solution. Game elements such as time restrictions and turn-taking are used to structure the game sessions.

User Involvement - The game eases communication about complex problems using simplified graphics and allows players to safely explore the solution space of various Urban Planning issues in a neighborhood planning project. Each player takes the role of a stakeholder (e.g. the mayor, an inhabitant or a housing corporation). Through this role, players are able to suggest and place solutions in the neighborhood (projected on the surface table) and see the effect. All players, as a team, have the opportunity to negotiate, discuss and decide which solution is most beneficial to the group (see figure 7). The primary aim of the game is not to 


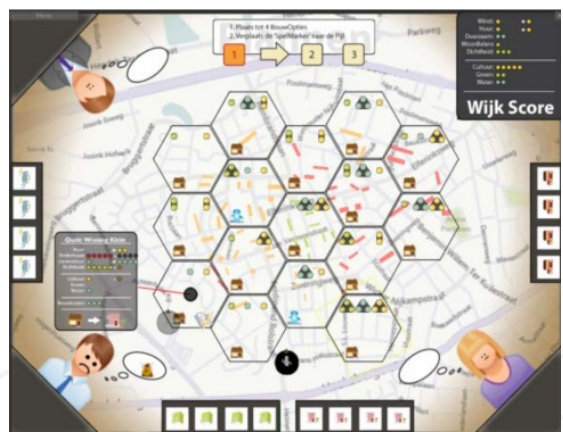

Figure 6. A top view of the urban planning game, showing a city region and three characters that play a role in the game.

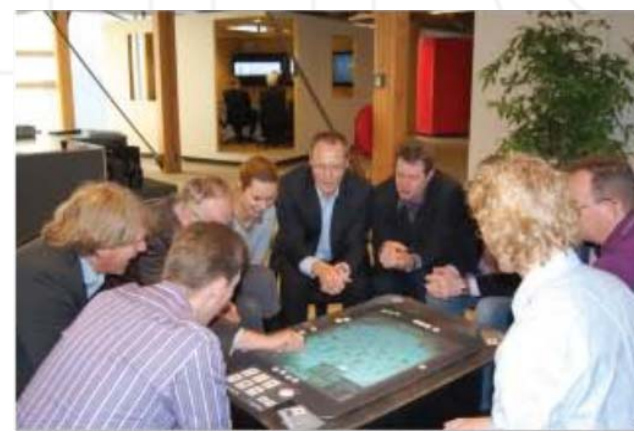

Figure 7. Participants in an Urban Planning game session.

directly generate solutions, but rather to trigger discussions and explore new possibilities in early stages of an urban planning project.

\subsection{Virtual usability lab}

Application Context - The Virtual Usability Lab was created during a case study for a manufacturer of professional office machinery (primarily printers). The company had already achieved some level of user involvement in the early stages of their design process by asking future clients to evaluate new graphical user interface (GUI) prototypes. However, the reliability of these evaluations is reduced by the fact the GUI concepts were not evaluated in a realistic use context; the GUI concept is not connected to a physical printer, so evaluation participants have to imagine what the interaction with the real machine would be like. The Virtual Usability Lab addresses this shortcoming by providing a realistic virtual use context in which virtual office machinery can be evaluated by end-users.

Technical Implementation - During the case study two technical implementations for the Virtual Usability Lab have been developed and evaluated with company participants. Both implementations include a virtual office environment in which existing and future virtual printers can be operated (e.g. paper refill, start/stop printing, etc.). The first implementation 
uses hand-held augmented reality tablets to visualise the interactive virtual environment. Pointing the AR tablet on specific AR markers allowed users to see and interact with virtual objects. The second implementation provides the user with a first-person interactive walkthrough on a wall display. The first implementation enables users to physically navigate the virtual office by moving between markers and pointing the AR tablet. The second implementation uses a more traditional interface, but does allow for a more immersive visualisation. After an evaluation of the two implementations (involving participants from the case study company) the fully virtual environment was considered more effective for representing the use context (see figure 8 and 9). While the augmented reality environment did allow for more physical interactions (e.g. really walking around an object), it failed to keep the designers 'immersed' in the virtual environment. The fully virtual environment was found to provide a more integrated experience.

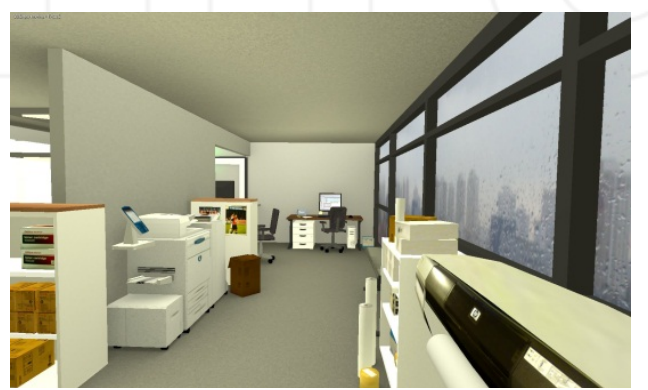

Figure 8. A screenshot of the first-person perspective of the virtual usability lab.

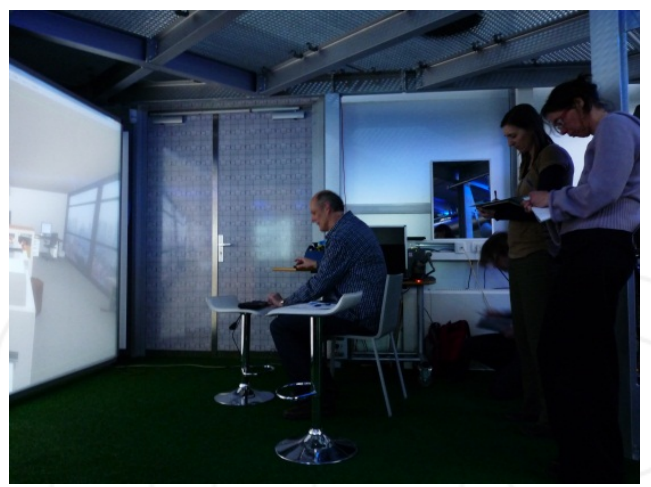

Figure 9. Designers (on the right) using the virtual usability lab in a test case.

User Involvement - The case study showed that the application primarily helps with analysing high-level task flows, such as operator movement between different machines or locations in the office. Being able to track and analyse these movements, as well as the actions that take place at each location adds a lot of useful knowledge to the early design phases. The presented application allows designers to capture this knowledge (i.e. by letting real operators act out a 'day at work' in a future virtual office), but also to communicate this 
knowledge to other designers, engineers or marketeers who are usually not aware of certain real-life situations. Physical analyses, such as testing out different positions of paper trays on a virtual printer, were found to be less useful. Unless the virtual environment would also simulate weight, friction and other tactile aspects it is usually more effective to evaluate those aspects using physical prototypes.

\subsection{Virtual nursery room}

Application Context - The Virtual Nursery Room case study comprised the design of a Neonatal Intensive Care Unit (NICU) patient area [18]. A NICU houses premature babies in incubators and includes a large number of medical appliances to monitor and nurse the newborn. The involvement of end-users in the design process of such a room is important, since particularly in medical design few designers are familiar with and can therefore anticipate the specific use situations of the product and the demands that arise from it. Neither can the emotional situation of the parents that have their child lying at the NICU be envisioned to the full extend by persons that have not experienced a similar situation. A participatory design approach was used to evaluate room design concepts in a very early stage of the project.

Technical Implementation - For an optimal evaluation with regard to both emotional and usability aspects, it is essential that users can actually experience the room designs. Therefore, it was chosen to conduct a participatory session in a mixed reality setting: In an evaluation session hospital staff engaged with virtual representations of the candidate designs and judged them on usability as well as on emotional impact. The concepts were presented on a screen as pictures and in three similar animations. Afterwards, rendered pictures of the designs were projected life-size on a concave screen. A simple physical table was placed in front of the projection, representing the incubator. A baby dummy was placed on the table, fitted with medical material (see figure 10).

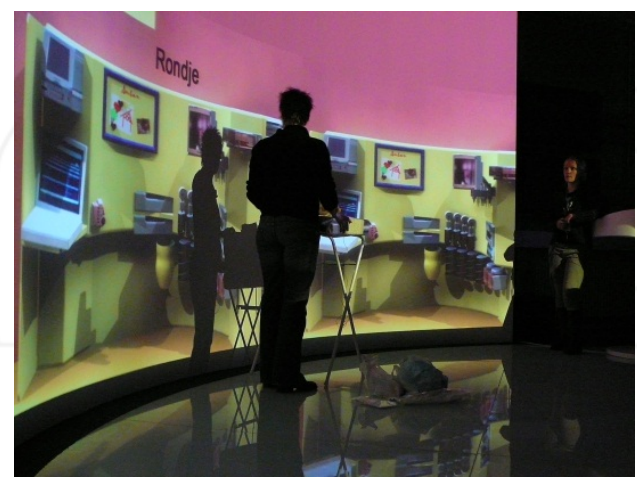

Figure 10. Participants acting out scenarios in front of a projected nursery room.

User Involvement - Participants, consisting of nurses and doctors were asked to play out nursing scenarios within the setup. The participants started with making a first concept 
choice based on how they estimated the emotional impact of the concepts. After playing out the nursing scenarios, the participants concluded that their first choice (based on emotional impact) had some usability issues. The case study illustrates how relatively simple mixed reality environments can help participants to better understand (future) situations, and review them from various perspectives. In this mixed reality sessions participants were able to evaluate concepts on both emotional and usability aspects.

\subsection{Haptic machine lid simulation}

Application Context - This case study aimed to demonstrate how VR can help with shifting design decisions regarding physical components to an earlier stage of the design process [19]. The case study features the design of the machine lid of which the hinges were to be chosen. The specification of these hinges (for instance their stiffness) affects the use experience of the whole machine. Instead of building several prototypes with various hinges a virtual prototype was created using a haptic feedback device.

Technical Implementation - For a realistic simulation of the lid hinges, an FCS-CS Haptic Master was used. A grip from a real machine was connected to the haptic arm. The haptic device was synchronised with a digital visualisation of the machine; moving the haptic device up and down allowed users and designers to open and close the virtual machine model (see figure 11). The haptic device could be configured to simulate various types of hinges.

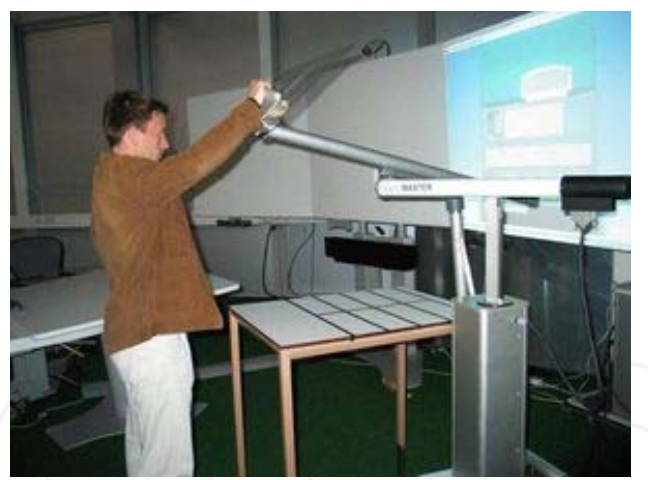

Figure 11. A designer testing the haptic lid emulator. The display on the right shows a digital representation of the machine that is linked to the haptic device.

User Involvement - Unlike most of the other case studies presented in this chapter, this case study focused on simulating physical product aspects. The use of the haptic device allows designers to efficiently and realistically evaluate various hinges.

\subsection{Operating theater design}

Application Context - This case study comprises the design and evaluation of a VR design tool for operating theaters [20]. The application supports stakeholders of dedicated endoscopic 
operating theaters, such as surgeons, nurses and anesthetists, with the design of a ceiling mounted arm (CMA) systems in a virtual operating theater environment and gain insight in consequences of design decisions.

Technical Implementation - The VR application consists of a Microsoft Surface Table, a large wall display and miniature physical models of the CMA systems. The Surface Table displays a top down view of an operating theater, in which participants can create CMA concepts by placing the miniature CMA models (see figure 12). The models correspond to digital CMA systems in the virtual environment, projected on the large wall display. The wall display allows participants to walk-through the operating theater while still modifying it on the Surface Table (see figure 13).

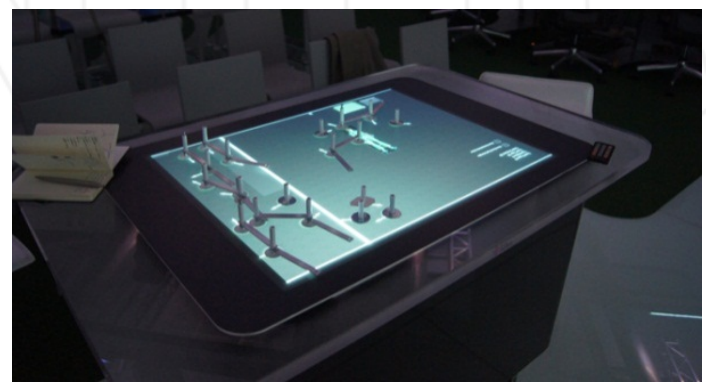

Figure 12. The Surface Table with tangible CMA (Ceiling Mounted Arm) systems used for the operating theater design case study.

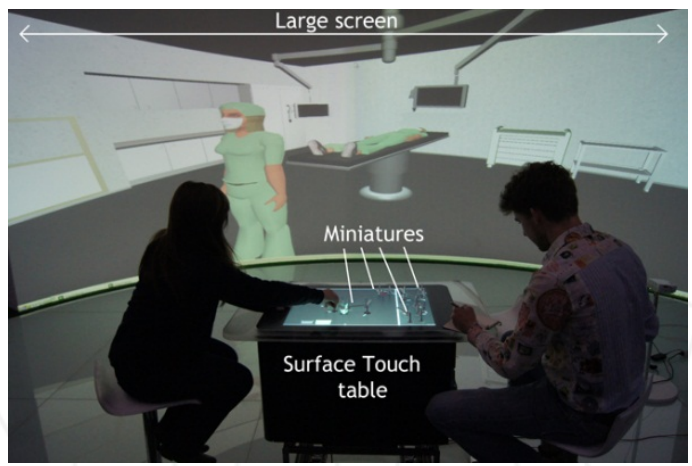

Figure 13. Operating theatre design; the surface table is used as an interface for the virtual theater (shown in the background)

User Involvement - The setup allows session participants to immerse into the environment by the use of movable virtual avatars and experience the consequences of the design decisions they have made. The effect of re-positioning a CMA system (which is difficult in real-life) is immediately visible for everyone on the wall display. As such the application can be used to evaluation CMA design concepts, but also to reenact use scenarios with future CMA systems, list priorities, etc. 


\subsection{ADAS feedback modality simulator}

Application Context - The design of advanced driver assistance systems faces the challenge of providing automated support behavior that complements the human driver safely and efficiently. Driving simulators can be used to test these new systems, as illustrated in the Lane-Change Support case study. However, developing accurate virtual prototypes of these systems is time consuming. This case study therefore investigated the use of human emulation as a simulation alternative (known as the Wizard of $\mathrm{Oz}$ approach) [21].

Technical Implementation - An automated and an emulated version of a lateral support system were compared in a fixed-base driving simulator setup. Test participants are given a driving task (e.g. follow a preceding car). During this task they are given a cue on which they have to respond. The driver support was either generated by a predefined automatic version or by a human co-driver that emulated the support behavior. Co-drivers were seated behind a curtain and drivers were unaware of their presence and task. Co-Drivers controlled a secondary steering wheel which was connected to the drivers' steering wheel.

User Involvement - The case study addresses the same topic as the Lane-Change Support System case study, but provides users with predefined designs rather than asking users to generate solution concepts themselves. As a result, the technical complexity of this setup is reduced. The case study revealed that the behavior of a future driver support system can effectively and realistically be programmed as a human operator instead of a virtual prototype of the system. Consequently this approach is considered very useful for early concept selection.

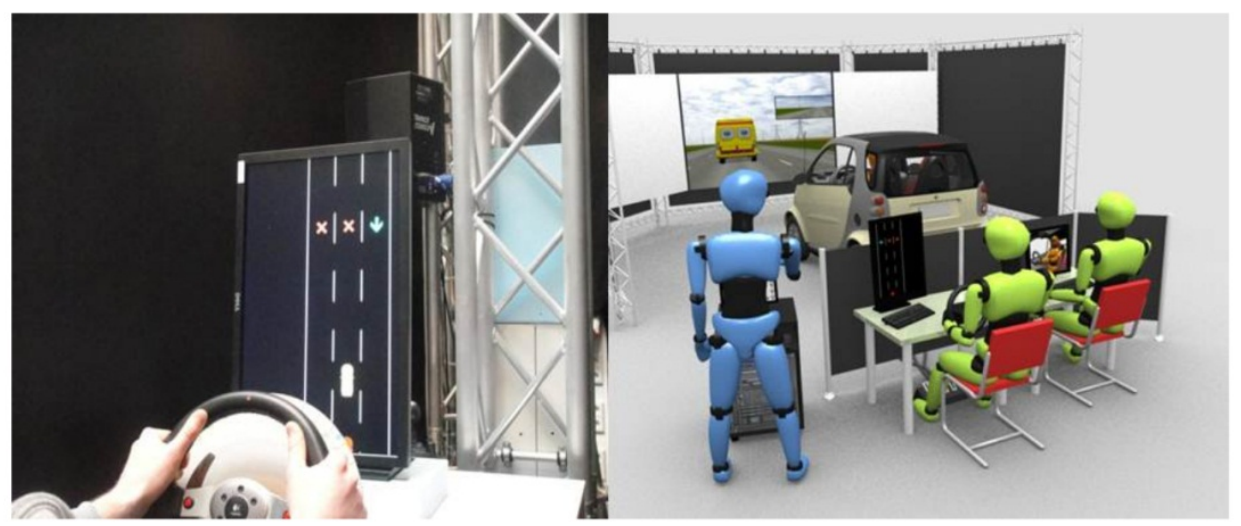

Figure 14. ADAS Feedback Modality Simulator: on the left, a human operator can send cues (e.g. haptic cue via steering wheel) to a test participant in the simulator (on the right)

\subsection{AR discussion table}

Application Context - The Augmented Reality (AR) Discussion Table was developed for a manufacturer of industrial dough processing machines. The client was interested in 
increasing end-user involvement in their product development process. The primary challenge in this case study was the communication between machine engineers, end-users (machine operators) and decision makers (customers). Especially when customers have to decide whether or not a proposed machine design meets their requirements it is sometimes difficult to reach an understanding between the stakeholders.

For this application an Augmented Reality (AR) table was developed to provide a more intuitive visualisation of machine design proposals. While the engineers currently use $2 \mathrm{D}$ technical drawings and sometimes renders of CAD models, the $\mathrm{AR}$ table provides an interactive $3 \mathrm{D}$ visualisation of an entire machine layout.

Technical Implementation - The AR Table uses an off-the-shelf web camera that is pointed on a table surface. The camera stream is sent to AR tracking software to identify and track specific markers. The position and orientation of each marker corresponds to a specific machine CAD model, resulting in a 3D interactive representation of the machine layout. Each machine can be rotated and re-positioned by moving the markers on the table, or by rotating the camera around the table. The entire 3D scene is projected on a large display next to the table.

User Involvement - Similarly to the Room Layout Configurator this application offers a lowthreshold multi-user interface for manipulating 3D scenes. The AR table can be used to support meetings between the engineers, sales department and potential customers, allowing all participants to change or inspect the machine setup.

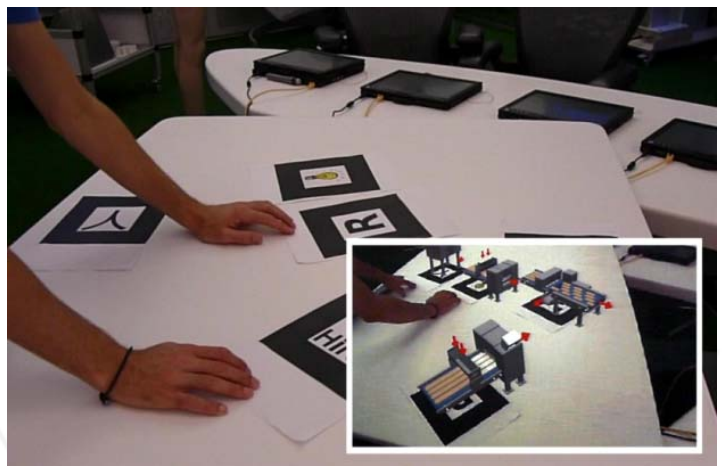

Figure 15. The Augmented Reality Table. The picture shows the markers and (inset) the virtual models projected on top of these markers.

\section{Review}

The overview of case studies illustrates the diversity of opportunities for VR applications in the early stages of the product development process and confirms the industrial need for this type of applications. Structuring the case studies according to their position in the PDP and the achieved level of user engagement provides several insights regarding the facilitation of user engagement through VR. 


\subsection{Application scope}

The majority of the presented VR applications have a relatively narrow scope, meaning that they target a particular phase in the early stages of the PDP. Only the development of entirely new complex systems (such as driver assistance systems) benefits from VR support throughout the first three phases of its development: virtual prototypes can elicit product requirements, can be tested and improved by end-users, and serve as an evaluation or validation platform in the final stages of concept design. In return for a relatively high investment this offers a safe, flexible and time efficient development approach. For redesign or incremental innovation projects on the other hand, VR can be particularly useful for evaluating multiple concept directions. For instance, the re-design of office machinery does not usually involve radical modifications, but would benefit from being able to quickly evaluate several minor modifications to existing products.

As explained in section 2 of this chapter, scenarios and gaming techniques proved to be useful supporting principles in several case studies. Scenarios have been used to embed a specific VR technology in a more extensive approach, as illustrated in the Office Machinery and the Aircraft Galley case studies; participants act out predefined scenarios in a virtual environment. The use of the virtual environment strengthens the realism of the scenarios, while the scenarios add 'meaning' to the virtual environment. In the Urban Planning Game game elements made 'wicked problems' manageable for non-expert stakeholders, for instance by using metaphors and simplified scoring systems.

Specifying a proper balance between VR, supporting principles and other tools and methods turns out to be case dependent. In the automotive case studies for instance, the VR simulation and evaluation platform forms the basis of the method. The NICU case study on the other hand shows a more traditional participatory design process of which VR is a relatively small part.

\subsection{User engagement}

While it is not possible to strictly allocate an application to a certain level of user engagement, the majority of the early stage VR applications facilitates evaluation tasks, and aims to 'design with' end-users. A higher level of user engagement is possible, but often requires technically complex VR applications. With this level of engagement, the VR application needs to provide an appropriate user interface and flexible modeling features. Furthermore, end-users may need additional training or instructions to participate. In the ADAS interface configuration case study for instance, participants received instructions on how to create their personal user interface. However, emerging interface technologies such as multi-touch displays allow for more intuitive interactions, making it easier for end-users to participate in generating or at least modifying product concepts, as shown in the case studies that used a Surface Table as input device. When using VR to support the lower level of user engagement, it is obvious that VR facilitates the design process, but the added value with respect to user engagement is usually less obvious compared to the higher levels of engagement. 


\section{Conclusion}

The case studies show how VR facilitates user involvement in various phases of the design process. While it should be noted that the review of case studies is currently limited to our own experience, the resulting insights could be valuable on a more generic level as well. The case studies were characterized based on the design phase application as well as the level of user engagement. The majority of the VR applications aims to 'design with users' in concept evaluations. From an industrial point of view, this type of application offers clear 'value for money'; they are not as complex as for instance the concept generation tools, yet offer clear benefits with respect to user engagement. Nevertheless, new interaction technologies more and more reduce the threshold for exploring concept generation and modification applications.

Successful deployment of VR in the early stages of a product development process is still very much case dependent. Incremental development seems to benefit from task specific applications while for new product development a wider scope of VR support could be worth the investment. Furthermore, supporting principles such as gaming techniques and scenarios can extend and strengthen the applicability of VR; VR can become part of a scenario based approach, or scenarios and gaming techniques can be used to give meaning to a VR application.

\section{Author details}

J.P. Thalen and M.C. van der Voort

Laboratory of Design, Production and Management, Faculty of Engineering Technology,

University of Twente, the Netherlands

\section{References}

[1] Pahl G, Beitz W (1984) Engineering Design: a Systematic Approach. Springer, Berlin, Germany.

[2] Suh N.P (1990) The Principals of Design. Oxford University Press, New York, USA.

[3] Sohlenius G (1992) Concurrent Engineering. Annals of the CIRP, Vol. 41, No.2, pp. 645655.

[4] Lu S.C.Y (2003) Engineering as Collaborative Negotiation: a New Paradigm for Collaborative Engineering Research. Whitepaper, Retrieved May 4th, 2006, from http://wisdom.usc.edu/ecn.

[5] Carroll J.M, Rosson M.B (1992) Getting around the task-artifact cycle: how to make claims and design by scenario. ACM Transactions on Information Systems, Vol. 10, pp. 181-212.

[6] Carroll J.M (2000) Five reasons for scenario based design. Interacting with Computers, Vol. 13, No. 1, pp. 43-60. 
[7] Tideman M, van der Voort M.C, van Houten F (2008) A New Product Design Method Based on Virtual Reality, Gaming and Scenarios. International Journal on Interactive Design and Manufacturing 2, no. 4 (October 1, 2008): 195-205.

[8] Biocca F, Levy M.R (1995) Communication in the Age of Virtual Reality. Lawrence Erlbaum Associates, Hillsdale, NJ, USA.

[9] Tideman M, van der Voort M.C, van Houten F (2006) Haptic Virtual Prototyping for Design and Assessment of Gear-shifts. In: ElMaraghy, H.A., ElMaraghy, W.H. (eds.), Advances in Design, Springer, Berlin, Germany, pp. 461-472.

[10] Miedema J, van der Voort M.C, Lutters D, van Houten F (2007) Synergy of Technical Specifications, Functional Specifications and Scenarios in Requirements Specifications. In: Krause, F.-L. (ed.). The Future of Product Development: Proceedings of the 17th CIRP Design Conference, pp. 235 - 246, Springer

[11] Brandt E (2006) Designing Exploratory Design Games: A Framework for Participation in Participatory Design? In: Proceedings of the ninth Participatory Design Conference 2006, ACM, Trento, Italy, pp. 57-66.

[12] Ehn P, Sjögren D (1991) From system descriptions to scripts for action. In: Greenbaum, J. and Kyng, M. (eds.), Design at Work: Cooperative Design of Computer Systems, Lawrence Erlbaum Associates, Hillsdale, NJ, USA.

[13] Kaulio M (1998) Customer, Consumer and User Involvement in Product Development: A Framework and a Review of Selected Methods. Total Quality Management 9, no. 1 (1998): 141.

[14] Jimeno A, Puerta A (2007) State of the art of the virtual reality applied to design and manufacturing processes. In: The International Journal of Advanced Manufacturing Technology, Vol. 33, No. 9, 866-874

[15] Tideman M, van der Voort M.C, van Houten F (2008) A New Product Design Method Based on Virtual Reality, Gaming and Scenarios. In: International Journal on Interactive Design and Manufacturing 2, no. 4 (October 1, 2008): 195-205.

[16] Thalen J, van der Voort M.C (2011) User Centred Methods for Gathering VR Design Tool Requirements. Joint Virtual Reality Conference of EGVE-EuroVR, 75-81, 2011.

[17] Miedema J (2010) Synthetic Environments in Design Processes. PhD thesis, University of Twente

[18] Garde J, van der Voort M.C (2009) The Design of a New NICU Patient Area: Combining Design for Usability and Design for Emotion.

[19] Miedema J, Meijer F, Wang H, van der Voort M, van den Broek E, Vergeest S (2007) Synthetic Environments as Design Tool - A Case Study. Article in monograph or in proceedings, 2007. http://doc.utwente.nl/58730/.

[20] Huijing S, Garde J (2011) Applying Virtual Reality for Participatory Design: supporting end-users in the design process of an endoscopic operating theatre. In: Norbert Roozenburg, Lin-Lin Chen and Pieter Jan Stappers (eds), Proceedings of IASDR2011, 
4th World Conference on Design Research, October 31 - November 4, 2011. TUDelft, 2011.

[21] Waterschoot B.M, van der Voort M.C (2012) Optimizing the design of driver support: Applying human cognition as a design feature. Proceedings of the $4^{\text {th }}$ International Conference on Applied Human Factors and Ergonomics, in press, CRC press / Taylor \& Francis.
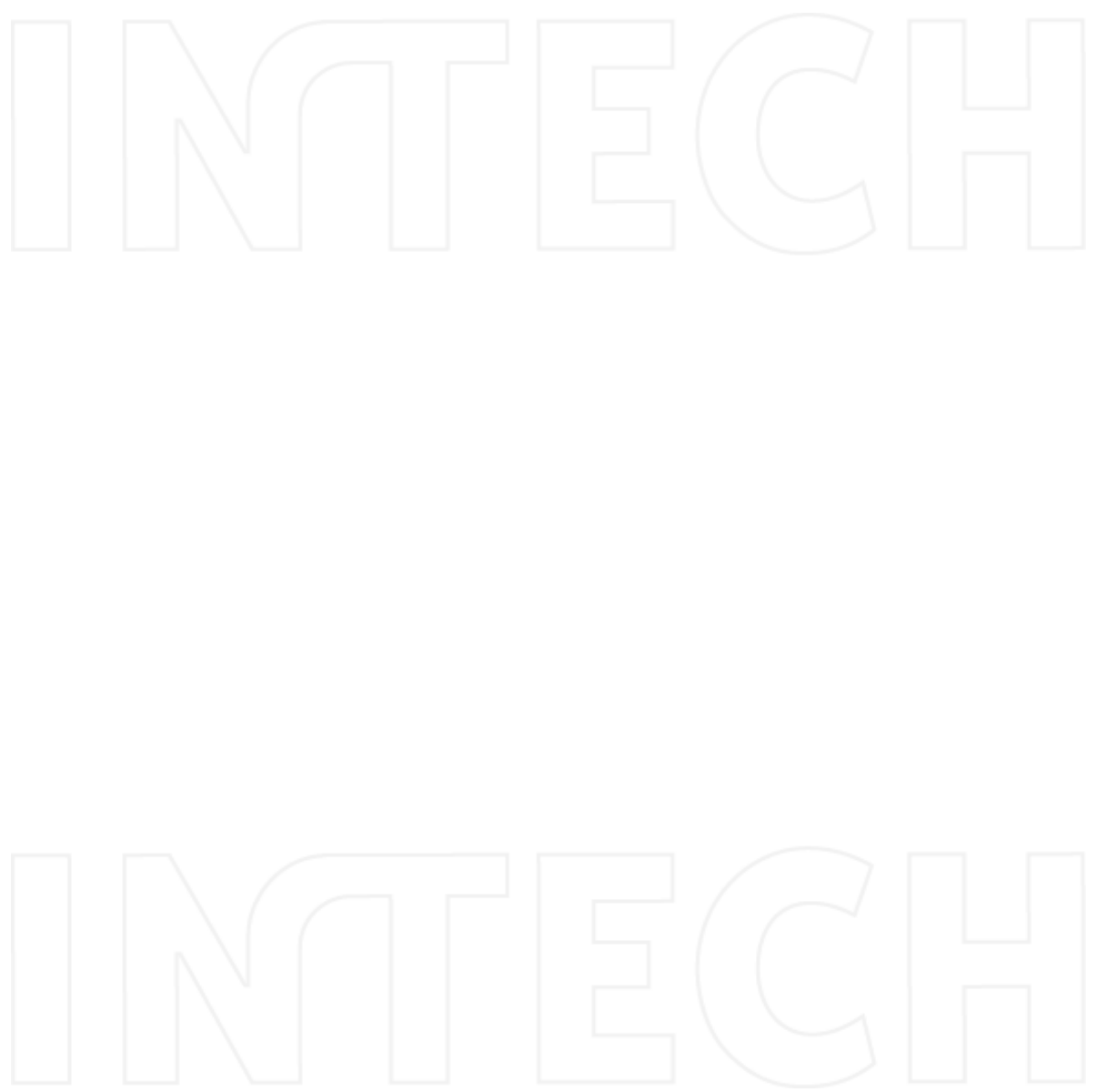